\title{
Mapping and Comparative Analysis of QTL for Crown Rust Resistance in an Italian $\times$ Perennial Ryegrass Population
}

\author{
S. Sim, K. Diesburg, M. Casler, and G. Jung
}

First author: The Ohio State University-OARDC, Department of Horticulture and Crop Science, 1680 Madison Ave., Wooster 44691 ; second author: Southern Illinois University-Carbondale, Department of Plant, Soil and Agricultural Systems Mailcode 4415, Carbondale 62901; third author: United States Department of Agriculture-Agricultural Research Service U.S. Dairy Forage Research Center, 1925 Linden Dr., Madison, WI 53706; and fourth author: University of Massachusetts, Department of Plant, Soil, and Insect Sciences, 230 Stockbridge Rd., Amherst 01003.

Accepted for publication 18 December 2006.

\begin{abstract}
Sim, S., Diesburg, K., Casler, M., and Jung, G. 2007. Mapping and comparative analysis of QTL for crown rust resistance in an Italian $\times$ perennial ryegrass population. Phytopathology 97:767-776.

Crown rust (Puccinia coronata $\mathrm{f}$. sp. lolli) is a serious fungal foliar disease of perennial ryegrass (Lolium perenne $\mathrm{L}$.) and Italian ryegrass $(L$. multiflorum Lam.), which are important forage and turf species. A number of quantitative trait loci (QTL) for crown rust resistance previously were identified in perennial ryegrass under growth chamber or greenhouse conditions. In this study, we conducted a QTL mapping for crown rust resistance in a three-generation Italian $\times$ perennial ryegrass interspecific population under natural field conditions at two locations over 2 years. Through a comparative mapping analysis, we also investigated the syntenic relationships of previously known crown rust resistance genes in other ryegrass germplasms and oat, and genetic linkage between crown rust resistance QTL and three lignin genes: LPOMT1, $L p C A D 2$, and $L p C C R 1$. The interspecific mapping population of 156 progeny was developed from a cross between two Italian $\times$ perennial ryegrass hybrids, MFA and MFB. Because highly susceptible reactions to crown rust were observed from all perennial ryegrass clones, including two grandparental clones and eight clones from different pedigrees tested in this study, two grandparent clones from Italian ryegrass cv. Floregon appeared to be a source of the resistance. Two QTL on linkage groups

regardless of year and location. The others, specific to year and location, were located on LGs 3 and 6 in the susceptible parent MFB map. The QTL on LG2 was likely to correspond to those previously reported in three unrelated perennial ryegrass mapping populations; however, the other QTL on LGs 3, 6, and 7 were not. The QTL on LG7 was closely located in the syntenic genomic region where genes $P c a$ cluster, Pcq2, $P c 38$, and Prq1b resistant to crown rust ( $P$. coronata f. sp. avenae) in oat (Avena sativa L.) were previously identified. Similarly, the QTL on LG3 was found in a syntenic region with oat genes resistant to crown rust isolates PC54 and PC59. This indicates that the ortholoci for resistance genes to different formae speciales of crown rust might be present between two distantly related grass species, ryegrass and oat. In addition, we mapped four restriction fragment length polymorphism loci for three key ryegrass lignin genes encoding caffeic acid- $O$-methyltransferase, cinnamyl alcohol dehydrogenase, and cinnamoyl CoA-reductase on LG7. These loci were within a range of 8 to 17 centimorgans from the QTL on LG7, suggesting no tight linkage between them. The putative ortholoci for those lignin biosynthesis genes were identified on segments of rice (Oryza sativa L.) chromosomes 6 and 8, which are the counterparts of ryegrass LG7. Results from the current study facilitate understanding of crown rust resistance and its relationship with lignin biosynthesis, and also will benefit ryegrass breeders for improving crown rust resistance through marker-assisted selection.
\end{abstract} (LGs) 2 and 7 in the resistant parent MFA map were detected consistently
Two primary species in the genus Lolium are perennial ryegrass (Lolium perenne L.) and Italian ryegrass (L. multiflorum Lam.), which are cultivated throughout the world. Perennial ryegrass is a valuable grass species used for forage and turf, while Italian ryegrass is used mainly for hay and silage and for establishing winter overseeding in warm-season turf. Both species are diploid $(2 n=$ $2 x=14$ ), outcrossing, and self incompatible. In these species, crown rust is a serious foliar disease caused by the biotrophic fungus Puccinia coronata f. sp. lolii. It is widespread in the temperate regions where perennial ryegrass or Italian ryegrass is cultivated. This disease decreases herbage yield, nutritional quality, and palatability to grass-eating domestic animals (21).

The development of resistant cultivars is an important objective in ryegrass breeding programs, because the use of resistant cultivars is the most reliable and environmentally sound option among several crown rust control strategies (21). Perennial ryegrass genotypes carrying genes resistant to crown rust have been identi-

Corresponding author: G. Jung; E-mail address: jung@psis.umass.edu

doi:10.1094/PHYTO-97-6-0767

(c) 2007 The American Phytopathological Society fied $(6,22,32)$. Further, genetic studies of crown rust resistance have revealed evidence for both qualitative and quantitative inheritance in perennial ryegrass $(12,26,32)$ and in Italian ryegrass (40). This indicates that the major genes or quantitative trait loci (QTL) for crown rust resistance exist in different ryegrass germplasms, which can be utilized for improving resistance in ryegrass through breeding programs.

Molecular marker-based linkage maps have facilitated the estimation of the location, number, and effect of QTL or major genes for disease resistance in plants $(8,10,29)$. Several linkage maps of ryegrass have been reported since the first map of Hayward et al. (13). These maps have employed various marker types such as amplified fragment length polymorphism (AFLP), random amplified polymorphic DNA (RAPD), restriction fragment length polymorphism (RFLP), and simple sequence repeat (SSR) $(20,33,36)$.

In perennial ryegrass, a number of QTL mapping studies for crown rust resistance have been conducted under growth chamber or greenhouse conditions $(10,29,35)$. Thorogood et al. (35) detected several QTL of crown rust resistance on linkage groups (LGs) 2, 5, and 7 in their genetic map of a perennial ryegrass population derived from two cultivars, Aurora and Perma. Dumsday et al. (10) identified a major QTL, designated as $L p P c 1$, 
on LG2 using a bulk-segregant analysis in a two-way pseudo-testcross population derived from two perennial ryegrass cultivars, Vedette and Victorian. A recent study of Muylle et al. (29) mapped four QTL ( $L p P c 1$ to $L p P c 4$ ) on LGs 1 and 2 using a mapping population derived from two perennial ryegrass genotypes, SB2 and TC1. Although disease evaluation under growth chamber or greenhouse conditions is a rapid and reliable method, it does not always correspond to evaluation under natural field conditions. In fact, the discrepancy between greenhouse and field evaluations has been found in QTL mappings of crown rust resistance in oat $(30,43,44)$.

Comparative genetic mapping using a common set of heterologous RFLP markers has characterized the ryegrass genome relative to the oat, rice, tall fescue, and wheat genomes by conserved synteny and large-scale chromosomal rearrangements $(1,19,33)$. These genome relationships can be useful to identify common or unique genes for a trait of interest between ryegrass and related grass species. In addition, the anchored RFLP markers employed by different ryegrass linkage maps can be utilized to compare the map locations of QTL or major genes for traits of interest detected from different germplasms. For example, the study of Dumsday et al. (10) suggested the presence of ortholoci for crown rust resistance between perennial ryegrass and oat based on the syntenic genome relationship and anchored RFLP markers. Muylle et al. (29) also revealed that one of their QTL corresponded to $L p P c 1$, which previously was identified by Dumsday et al. (10).

In smooth bromegrass (Bromus inermis Leyss.), crown rust resistance was significantly correlated with high lignin content, which is not desirable for herbage digestibility (9). Also, the inhibition of enzymes involved in lignin biosynthesis increased susceptibility of wheat (Triticum aestivum) to stem rust $(P$. graminis f. sp. tritici) (28). However, the correlation between crown rust resistance and lignin content has not been studied in ryegrass. Recently, on LG7 in the p150/112 perennial ryegrass reference map (19), Cogan et al. (7) identified a number of QTL for herbage quality traits which are closely linked to three RFLP loci for the key lignin genes LPOMT1, LpCAD2, and LpCCRI encoding caffeic acid- $O$-methyltransferase (OMT), cinnamyl alco- hol dehydrogenase (CAD), and cinnamoyl CoA-reductase (CCR), respectively $(15,24,25)$.

The objectives of this study were to (i) identify QTL for crown rust field resistance in an interspecific (Italian $\times$ perennial) ryegrass population under diverse and natural field conditions, (ii) compare the QTL with those previously detected in the growth chamber and greenhouse studies in perennial ryegrass populations, and (iii) investigate the syntenic relationships of the resistance genes to different formae speciales of crown rust, and (iv) explore genetic linkage between the QTL for crown rust field resistance and three lignin genes, LpOMT1, LpCAD2, and LPCCR1.

\section{MATERIALS AND METHODS}

Plant materials. An interspecific ryegrass mapping population of 156 progeny individuals was derived from a cross between two highly heterozygous ryegrass clones, MFA and MFB (36). These parental clones are Italian $\times$ perennial ryegrass hybrids that were derived from separate crosses between two clones (Flor-1 and Flor-3) of the Italian ryegrass cv. Floregon and two clones (Manh1 and Manh-3) of the perennial ryegrass cv. Manhattan. Further details on the development of this population are described by Warnke et al. (36).

Four different clones (Flor-2, -4, -6, and -7) of Floregon were established from the same seedlot that was used to generate the original grandparental clones of the mapping population, because the exact grandparental clones were not available for this study due to their annual nature. Additionally, eight perennial ryegrass clones with various genetic backgrounds were used as check plants. Pedigrees of these plants are described in Table 1.

Field evaluation of crown rust resistance. Each progeny of the mapping population was clonally divided and transferred in conical pots (RLC-7 Super "Stubby" Cell; Stuewe and Sons Inc., Corvallis, OR) filled with soilless potting media (Scott's MetroMix 366-P). The plants were grown for 4 weeks in a greenhouse and then transplanted to two experimental plots at the O. J. Noer Turfgrass Research and Education Facility (OJN) in Verona, WI and the Southern Illinois University Turf Research and Education

TABLE 1. Mean crown rust reaction of mapping parents, grandparents, and check clones

\begin{tabular}{|c|c|c|c|}
\hline \multirow[b]{2}{*}{ Description, plant name } & \multicolumn{3}{|c|}{ Rating $^{y}$} \\
\hline & 04-WI & 05-WI & 04-IL \\
\hline \multicolumn{4}{|c|}{ Perennial ryegrass mapping grandparents } \\
\hline \multicolumn{4}{|l|}{ Italian ryegrass clones ${ }^{\mathrm{z}}$} \\
\hline Flor-2 & $0.0 \pm 0.0$ & $\ldots$ & $0.0 \pm 0.0$ \\
\hline Flor-4 & $0.0 \pm 0.0$ & $\ldots$ & $0.0 \pm 0.0$ \\
\hline \multicolumn{4}{|l|}{ Mapping parents } \\
\hline MFA & $0.6 \pm 0.7$ & $1.0 \pm 0.4$ & $2.0 \pm 0.0$ \\
\hline MFB & $3.7 \pm 1.2$ & $3.8 \pm 1.0$ & $5.0 \pm 0.0$ \\
\hline \multicolumn{4}{|c|}{ Perennial ryegrass cv. SR 4000 or SR 4500 clones } \\
\hline SR $4400-2$ & $4.4 \pm 0.8$ & $3.7 \pm 1.0$ & $\ldots$ \\
\hline SR4500-1 & $5.0 \pm 0.0$ & $4.8 \pm 0.5$ & $\ldots$ \\
\hline SR4500-2 & $5.0 \pm 0.0$ & $4.7 \pm 1.0$ & $5.0 \pm 0.0$ \\
\hline P8 & $4.9 \pm 0.3$ & $4.6 \pm 0.8$ & $5.0 \pm 0.0$ \\
\hline P9 & $4.8 \pm 0.7$ & $4.8 \pm 0.5$ & $5.0 \pm 0.0$ \\
\hline
\end{tabular}

y Ratings based on a scale of 1 to 5 , where $0=$ little or no visible reaction; $1=$ no visible sporulation, but abundant chlorotic flecks; $2=$ small pustules with chlorotic surrounding tissue; $3=$ small to medium pustules with chlorotic surrounding tissue; $4=$ medium to large pustules with little or no chlorosis; and $5=$ abundant large pustules. A rating is indicated by year (2004 [04] or 2005 [05]) and location (Wisconsin [WI] or Illinois [IL]) and is a mean disease score over two ratings in $04-\mathrm{WI}$ and four ratings in $05-\mathrm{WI} ; \ldots=$ not determined.

${ }^{\mathrm{z}}$ Established from same seedlot that generated Italian ryegrass mapping grandparents. 
Center (SIU) in Carbondale, IL. The OJN area has much cooler temperatures and a shorter photoperiod compared with the SIU area during the growing season of ryegrass. The soil at the SIU area is a Hosmer Silty Clay Loam with a $\mathrm{pH}$ of 6.2 , whereas that of the OJN area is a Silty Loam with a $\mathrm{pH}$ of 6.6. The size of experimental plots at both locations was 15 by $24 \mathrm{~m}$. For the OJN plots, circles $0.45 \mathrm{~m}$ in diameter with spacing of $0.45 \mathrm{~m}$ from center to center were created by applying a solution of $4 \%$ Roundup (glyphosate) to an area which was covered by a mixture of perennial ryegrass and Kentucky bluegrass. An individual progeny of the mapping population then was transplanted in each circle.

For the SIU plot, individual progeny were transplanted with spacing of $0.45 \mathrm{~m}$ from plant to plant in a tilled area with no vegetation. In May 2004, 152 of the original 156 progeny were transplanted to both the OJN and SIU plots. In 2005, we reestablished an experimental plot at OJN because a large number of plants established in 2004 did not survive due to severe winter kills. Also, a new experimental plot was established at SIU in 2005. For both new experimental plots, only 143 progeny were transplanted at each site due to plant mortality of certain genotypes. The mapping parents, grandparents, and eight check perennial ryegrass clones also were included with the progeny of the mapping population. A randomized complete block design was used with five replicates of each genotype at each site. After transplanting, the plants were fertilized once and mowed once a week at a mowing height of $7.6 \mathrm{~cm}$, which maintained them in vegetative growth. During crown rust season from July to September, the plants were not mowed because crown rust epidemics can be prevented by mowing. Average height of the plants was approximately $20 \mathrm{~cm}$ at the first rating date and only seven progeny produced seed stalks.

Crown rust epidemics occurred by natural inoculation in 2004 and 2005 at both locations. The identity of crown rust was confirmed by the morphological characteristics of urediniospores and teliospores from 50 infected plants collected randomly throughout the plot. However, the presence of different physiological races for these natural inoculums was not investigated in this study. When large pustules were clearly seen on the leaves of the most susceptible perennial ryegrass clones SR4500-1 and SR4500-2, the progeny of the mapping population and other clones were visually rated for their reaction types to crown rust using the modified rating scale of Thorogood et al. (35): $0=$ little or no visible reaction; 1 = no visible sporulation, but abundant chlorotic flecks; 2 = small pustules with chlorotic surrounding tissue; $3=$ small to medium pustules with chlorotic surrounding tissue; $4=$ medium to large pustules with little or no chlorosis; and $5=$ abundant large pustules. In 2004, two ratings were conducted on 28 July and 11 August whereas, in 2005, four ratings were conducted on $2,9,16$, and 23 August at the OJN plots. At the SIU plot, one rating was conducted on 21 September 2004, but no rating was conducted in 2005 because a large number of plants did not survive after transplanting due to severe drought and heat stress in 2005.

Statistical analysis. Analysis of variance (ANOVA) for crown rust reaction type data was conducted using the General Linear Model (Proc GLM) procedure of SAS (SAS Institute, Inc., Cary, NC). A random model for all effects was used to analyze the seven experimental data sets (two ratings in 2004 and four ratings in 2005 at the OJN plots, and one rating in 2004 at the SIU plot) from the MFA/MFB population. Among a total of 152 progeny, 130 were chosen for this analysis due to missing data. Specifically, if more than two replicates of a progeny within each of the seven data sets were missing, it was necessary to exclude that progeny for the analysis. Broad sense heritability was determined over the seven ratings using the variance components calculated from expected mean squares from the ANOVA. In addition, phenotypic pairwise correlation was analyzed over all seven ratings using JMP software (SAS Institute, Inc.).
QTL analysis. Existing linkage maps of each parent, MFA and MFB $(33,36)$, were enhanced using additional 16 RFLP probes which were derived from creeping bentgrass cDNA, named 'Ast,' described by Chakraborty et al. (4). These marker data were collected from a progeny set of the same 89 individuals used by Sim et al. (33). In addition, RFLP probes for three ryegrass lignin genes ( $L p O M T 1, L p C A D 2$, and $L p C C R 1$ ) were generated by polymerase chain reaction (PCR) amplification of genomic DNAs of two parents, MFA and MFB.

For designing the primers of these lignin genes, their fulllength cDNA or genomic sequences were obtained from the National Center for Biothechnology Information GenBank: cDNA sequence for LPOMT1 (accession no. AF033538), cDNA sequence for $L p C A D 2$ (accession no. AF472592), and genomic sequence for LpCCRI (accession no. AY061889). Based on these sequences, a number of primer pairs were designed to amplify the fragments of those genes with sizes ranging from 400 to $1,000 \mathrm{bp}$. Once a primer pair produced a single clear band in each lignin gene, this PCR product was cloned and sequenced in order to validate whether it corresponded to the original sequence of the respective lignin gene.

The marker data for those lignin gene loci were collected from a total of 152 progeny, including a subset of 89 progeny used by Sim et al. (33) in order to define accurate map location of those loci. The linkage maps were constructed using the doubled-haploid population type option in JoinMap (Biometris, Wageningen, the Netherlands) and followed by Sim et al. (33).

The QTL analysis was conducted using MapQTL software (Biometris). Two parent maps were used separately to analyze each of seven ratings due to the unequal rates of recombination observed in the MFA and MFB maps (36) and due to the identification of QTL which were present in each parent map completely independent of the other parent map. The phenotypic variable for the QTL analyses was the mean score of reaction type over five clonal replicates of 89 progeny that were used for constructing the parent maps. Using interval mapping, significant QTL were identified by map location and phenotypic effect (the percentage of phenotypic variance explained). To determine the significant genome-wise logarithm of odds (LOD) threshold, a permutation test with 1,000 iterations was performed for each combination of ratings and parent maps. A significant QTL was claimed if it had a higher LOD score than the genome-wide threshold.

A nonparametric, single marker-based, Kruskal-Wallis analysis was conducted to confirm the significance of QTL detected by interval mapping, and to detect additional potential QTL when they were examined individually. If a genomic region was consistently significant at $P=0.01$ via the Kruskal-Wallis analysis, which might lead to the detection of false positives, it was still claimed as a QTL in order to prevent type II errors $(2,8)$ despite an LOD score lower than the genome-wise threshold. In addition, the phenotypic means of the two marker allele classes ' $a$ ' and ' $b$ ', present in each parental map, were calculated using this method for comparing actual mean values between the allele classes.

Once the significant QTL were detected using interval mapping and Kruskal-Wallis analyses, they were tested as a possible cofactor using the automatic cofactor selection option in MapQTL. Multiple-QTL model (MQM) mapping then was performed to detect other segregating QTL for crown rust resistance $(17,18)$.

Comparative analysis of crown rust resistance genes. The QTL and major genes for crown rust resistance detected in ryegrass and oat were compared in terms of their map location using common RFLP markers and the syntenic genome relationships $(19,33)$. In detail, Thorogood et al. (35) identified six QTL on LGs 2,5 , and 7 in their perennial ryegrass map. Because only the LG2 was aligned with our LG2 using common RFLP markers, a QTL on the LG2 was used for the comparative analysis in this study. Also, QTL designated as LpPcl to LpPc4 detected by 
Dumsday et al. (10) and Muylle et al. (29) were compared with our QTL because their map locations were known in the p150/112 perennial ryegrass reference map (19), which is comparable to our linkage map (33).

The resistance genes for oat crown rust used in this comparative analysis are $P c 38, P c a$ cluster, Prq1b, and $P c q 2$. The genes resistant to oat crown rust isolates PC54 and PC59 also were included. Pc38 and the genes resistant to crown rust isolates PC54 and PC59 were identified from two different hexaploid oat populations: the Pendek4838 population for Pc38 (37) and the Kanota/Ogle population for the other two genes (3). The Pca cluster was identified using a diploid oat population (Avena strigosa $\times$ A . wiestii) $(23,42)$. The QTL Prqlb and Pcq2 were detected from two hexaploid populations: the MN841801-1/Noble-2 population for Prqlb (30) and the Ogle/MAM17-5 population for Pcq2 (43,44).

\section{RESULTS}

Phenotypic evaluation of crown rust resistance. Crown rust evaluations at the OJN plots in 2004 and 2005 and at the SIU plot in 2004 showed similar patterns of disease reaction on the mapping parents, grandparents, and eight perennial ryegrass check clones. The perennial ryegrass grandparents, Manh-1 and Manh-3,
A

LG1
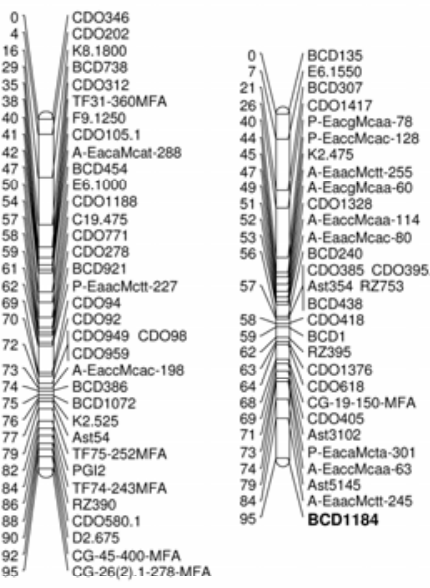

B

LG1

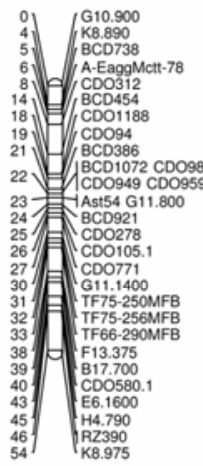

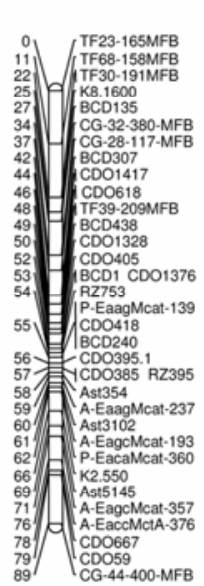

LG2

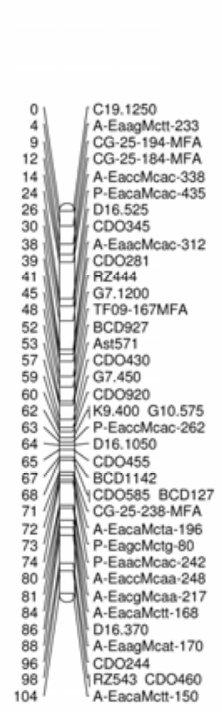

LG3

LG3

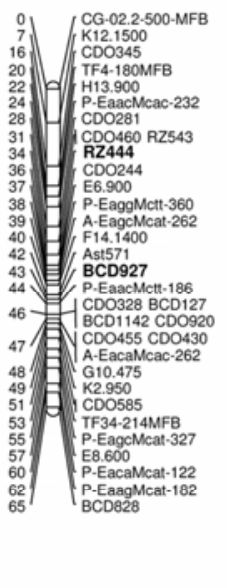

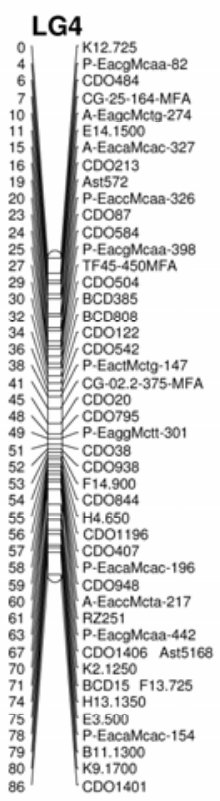

LG5

LG5

LG7

LG4

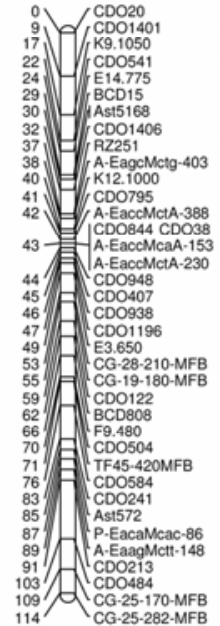

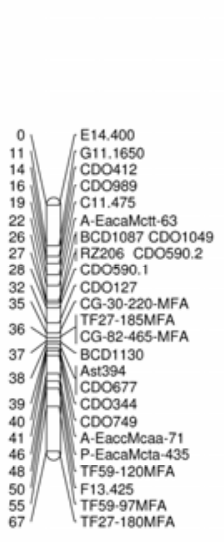

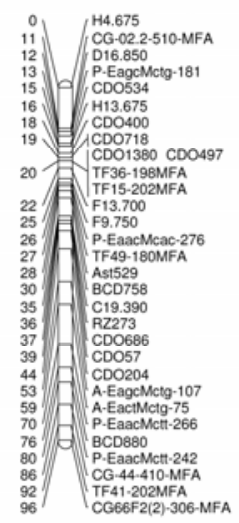

LG5

LG6

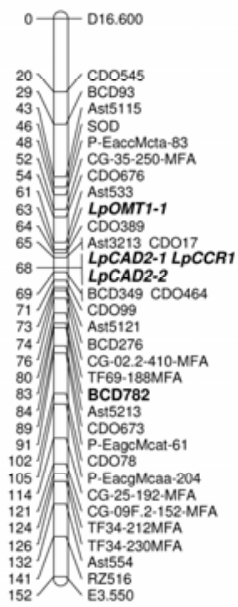

LG7
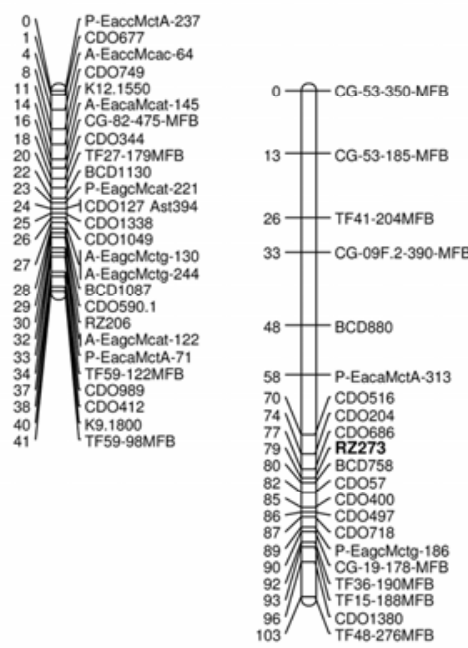

Fig. 1. Linkage maps of the MFA $\times$ MFB population used for quantitative trait loci (QTL) mapping for crown rust resistance in this study, constructed separately using the amplified fragment length polymorphism, random amplified polymorphic DNA, restriction fragment length polymorphism, simple sequence repeat, isozyme, and morphological marker data from Warnke et al. (36) and Sim et al. (33). Linkage groups for both maps are numbered according to Warnke et al. (36). A, MFA parent map and B, MFB parent map. The markers associated with the QTL detected in this study are shown in bold. Four loci for the lignin genes LPOMT1, LPCAD2, and LPCCR1 are shown in bold italics. 
showed highly susceptible reactions with large pustules, ranging from reaction types 4 to 5 (Table 1 ). The eight check plants, perennial ryegrass clones derived from the different pedigrees, also showed highly susceptible reactions similar to those observed in the perennial ryegrass grandparent clones (Table 1). However, the four different clones of Italian ryegrass cv. Floregon showed highly resistant reactions with no or chlorotic flecks, ranging from reaction types 0 to 1 for the mean value of five replicates throughout a period of all rating dates. Mapping parents MFA and MFB showed significantly and consistently different reactions to crown rust over locations, rating dates, and years. Resistant reactions ranging from reaction types 0 to 2 were observed on MFA, whereas susceptible reactions ranging from reaction types 2 to 5 were observed on MFB (Table 1).

The progeny of the mapping population showed relatively continuous distributions for their reactions to crown rust over seven ratings, but the frequency distribution from each rating seemed to have a different pattern as the disease progressed. The frequency distributions for the first rating in 2004 and 2005 at the OJN plots appeared to be skewed toward resistant reaction types, whereas the second rating in 2004 and the fourth rating in 2005 showed the frequency distribution skewed toward susceptible reactions. In addition, one rating at the SIU plot in 2004 showed the frequency distribution so highly skewed toward susceptible reactions that reaction types 0 to 1 for the mean value of five replicates were not observed in the progeny. Although each rating has a different pattern of frequency distribution, transgressive segregation was observed in all ratings, more obvious in earlier ratings than later ratings.

The ANOVA for all seven ratings over two locations in 2004 and 2005 showed significant effects for all factors, including genotype, genotype $\times$ location, genotype $\times$ year, and genotype $\times$ rating within year. Therefore, an individual data set from seven ratings was used separately for QTL analysis. The broad sense heritability based on variance component over 2 years and two locations was estimated to be 0.56 .

Strong phenotypic correlations were found among six ratings at the OJN plots over 2 years, with the coefficients ranging from
0.80 to 0.94 , while one rating at the SIU plot showed significant but weaker correlation with each of the six ratings at the OJN plots, with coefficients ranging from 0.64 to 0.69 .

QTL mapping of field resistance to crown rust. The MFA map covered a total map distance of 695 centimorgans (cM), with a total of 262 loci, whereas the MFB map covered a total map distance of $535 \mathrm{cM}$, with a total of 237 loci (Fig. 1A and 1B). The MFA and MFB maps contained 15 and 11 creeping bentgrass Ast markers, respectively, which were not previously employed by Sim et al. (33).

Based on these parent maps, four QTL for crown rust resistance were detected in this study (Table 2). For the ratings at the OJN plots, a significant QTL was identified consistently on LG2 of the MFA map over 2 years (Table 2). In 2004, this QTL was detected at both first and second ratings by interval or MQM mapping, and significant $(P \leq 0.005)$ by Kruskal-Wallis analysis. In 2005, although this QTL showed significant LOD scores $(>2.7)$ at only the first rating by both interval mapping and MQM mapping, it was significant $(P \leq 0.01)$ at the first, second, and fourth ratings by Kruskal-Wallis analysis. Total phenotypic variation explained by this QTL ranged from 7.8 to $15.3 \%$. An RFLP marker, BCD1184, was most closely associated with this QTL, and the ' $a$ ' allele of this marker appeared to be associated with crown rust resistance (Table 2). This resistant 'a' allele was absent in Manh-1, a perennial ryegrass parent of MFA.

A second QTL was identified consistently on LG7 of the MFA map for both years at the OJN plots (Table 2). For this QTL, the LOD scores exceeded genome-wide thresholds at the first and second rating in both 2004 and 2005 in MQM mapping. Further, this QTL was significant $(P \leq 0.005)$ over all six ratings by Kruskal-Wallis analysis. The percentage of phenotypic variance explained by this QTL ranged from 12.2 to $18.7 \%$. This QTL was most closely linked to RFLP marker BCD782, and the ' $b$ ' allele of this marker was associated with crown rust resistance. This resistant allele was absent in Manh-1.

In addition, a QTL on LG3 of the MFB map was noticed from the second rating at the OJN plot in 2004 (Table 2). This QTL was significant $(P \leq 0.005)$ by Kruskal-Wallis analysis even

TABLE 2. Summary of the quantitative trait loci (QTL) for crown rust resistance (reaction type) from seven rating dates at O. J. Noer Turfgrass Research and Education Facility in Verona, WI and the Southern Illinois University Turf Research and Education Center in Carbondale, IL in 2004 and 2005

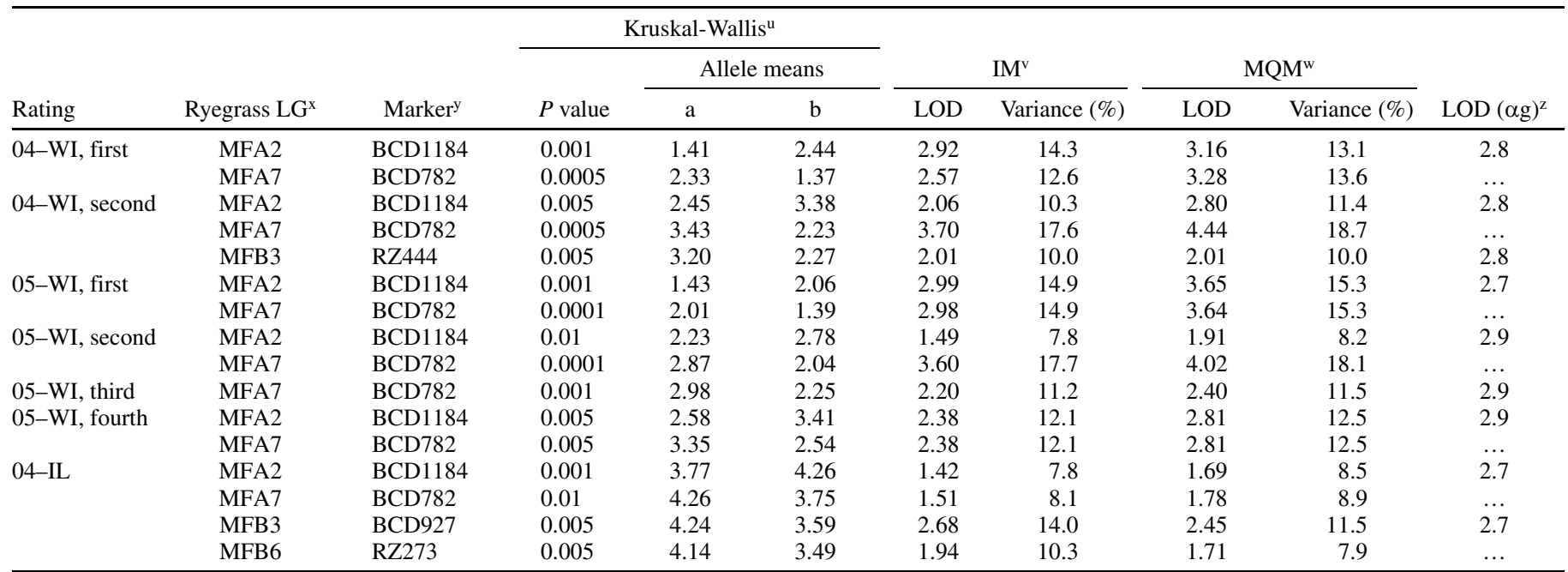

" For Kruskal-Wallis analysis, the $P$ value at the indicated marker is presented, along with mean phenotypic score of progeny carrying the 'a' or 'b' allele of each marker.

${ }^{v}$ For interval mapping (IM), the logarithm of odds (LOD) score and percentage of phenotypic variance explained at each of the named markers are given.

${ }^{\mathrm{w}}$ For multiple QTL model (MQM), the LOD score and percentage of phenotypic variance explained at each of the named makers are given. Also, the named markers were used as cofactors, after being selected for use as cofactors in the automatic cofactor selection test $(P<0.02)$.

${ }^{\mathrm{x}}$ Linkage group (LG) of parental map (MFA or MFB) in which each QTL was detected.

${ }^{y}$ Name of marker most closely linked to the QTL in question.

${ }^{\mathrm{z}}$ LOD thresholds derived from permutation analysis, for each combination of rating and parent linkage map, are given. The $\alpha \mathrm{g}$ indicates the 5\% LOD thresholds for all seven linkage groups of the parental map in question. 
A

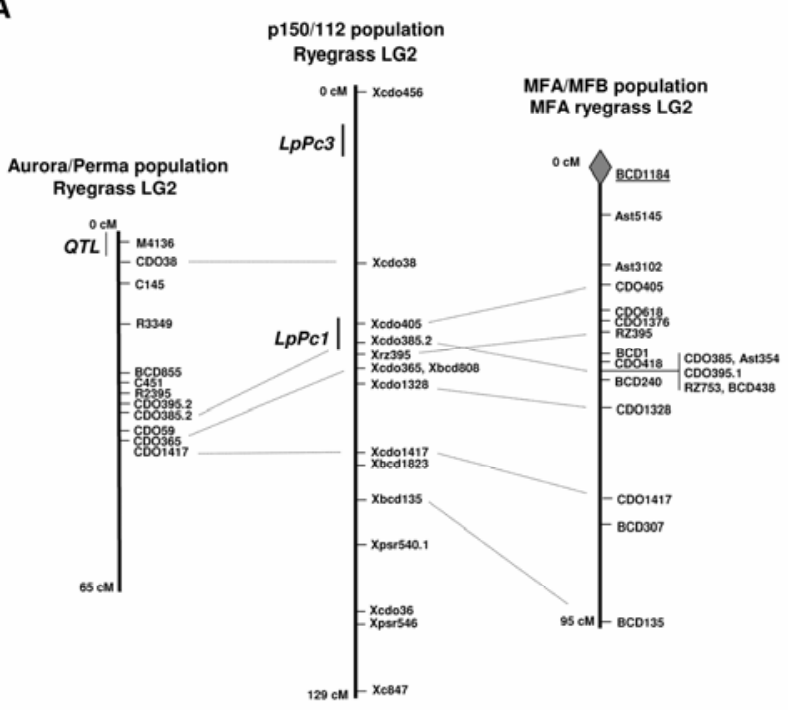

B

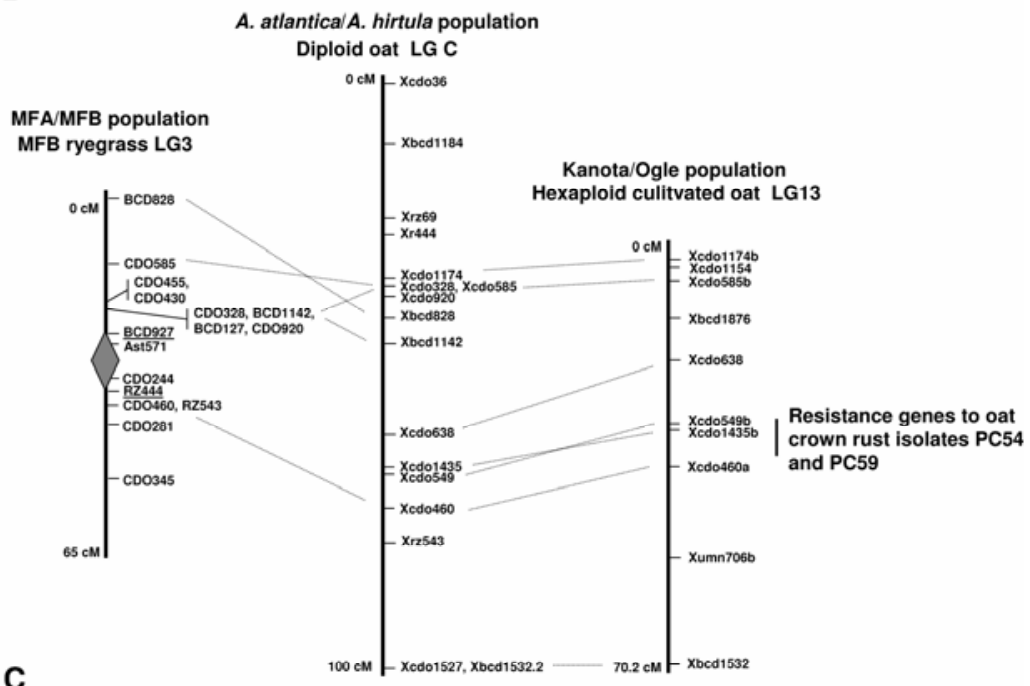

C

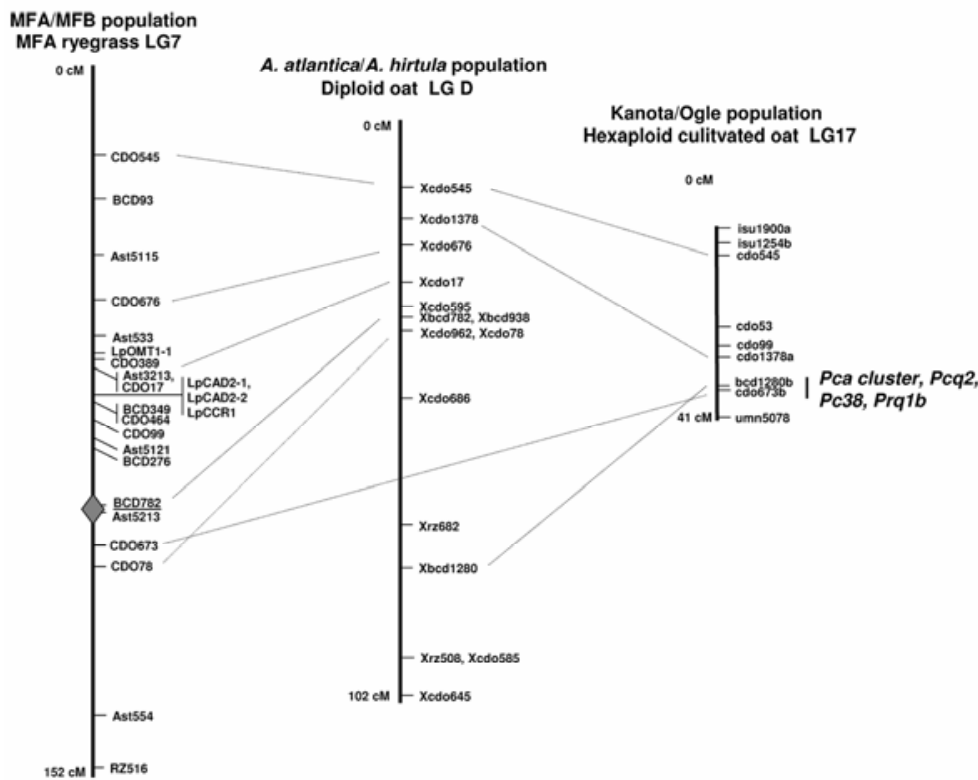

Fig. 2. Comparative mapping of quantitative trait loci (QTL) and major genes for crown rust resistance in ryegrass and oat. The locations of QTL detected in this study are indicated by a diamond shape on each linkage group (LG) of MFA $\times$ MFB population and most significant markers associated with the QTL were underlined. Approximate locations of the previously reported QTL and major genes are indicated by vertical lines with gene names in each LG. The dotted lines connect RFLP markers generated by the same probes and mapped on between LGs. A, Comparative relationship of the QTL on ryegrass LG2; B, comparative relationship of the QTL on ryegrass LG3; and $\mathbf{C}$, comparative relationship of the QTL on ryegrass LG7. 
though the LOD score (2.01) of the QTL, based on both interval mapping and MQM mapping analysis, did not exceed the genome-wide threshold (2.8). The percentage of phenotypic variation explained by RFLP marker RZ444 linked to the QTL was $10 \%$ (Table 2). The 'a' allele of this marker was associated with crown rust resistance and absent in Manh-3, a perennial ryegrass parent of MFB.

QTL analysis with one rating at the SIU plot in 2004 identified the same three QTL on LGs 2, 3, and 7 as identified at the OJN plots (Table 2). However, these QTL were significant $(P \leq 0.01)$ by Kruskal-Wallis analysis but not by interval or MQM mapping. For the QTL on LG3 of the MFB map, RFLP marker BCD927 likely was linked to the same QTL as RZ444 because the map distance between these two markers was $9 \mathrm{cM}$. In addition to the three QTL, an additional QTL, which was not detected in any of the ratings at the OJN plots, was noted on LG6 of the MFB map by Kruskal-Wallis analysis (Table 2 ).

Comparative mapping of crown rust resistance genes in ryegrass and oat. The QTL on LG2 of the Aurora/Perma perennial ryegrass population (35) was associated with an SSR marker, M4136, which is $\approx 5 \mathrm{cM}$ distant from RFLP marker CDO38 (Fig. 2A). Although this RFLP marker was not mapped on LG2 in the MFA map, its relative map distance from BCD1184, which is most closely linked to our QTL on LG2, was estimated based on common RFLP marker CDO405. The marker CDO38 was mapped with $\approx 15 \mathrm{cM}$ distance from Xcdo405 (CDO405) on LG2 in the p150/112 reference map (Fig. 2A). Because the distance between CDO405 and BCD1184 was $26 \mathrm{cM}$ on our LG2, the relative distance between $\mathrm{CDO} 38$ and $\mathrm{BCD} 1184$ could be $\approx 41 \mathrm{cM}$. The map locations of $\mathrm{LPCl}$ and $\mathrm{LpPc} 3$ were in the proximity of RFLP markers Xcdo385 (CDO385) and Xcdo456 (CDO456), respectively, on LG2 in the p150/112 reference map (Fig. 2A). The marker CDO385 was located $\approx 38 \mathrm{cM}$ distant from BCD1184 (Figs. 1A and 2A). In contrast, the accurate, relative map distance between BCD1184 and CDO456 could not be estimated due to lack of common markers between those ryegrass maps.

Two genes resistant to oat crown rust isolates PC54 and PC59 were located on LG13 of the Kanota $\times$ Ogle hexaploid oat map (3). This hexaploid oat LG13 is homoeologous with LG C of the A. atlantica $\times$ A. hirtula (Aah) diploid oat map (38). Additionally,
Jones et al. (19) and Sim et al. (33) have revealed the syntenic relationship between this diploid oat LG C and ryegrass LG3. Based on this syntenic relationship, the equivalent locations of oat genes resistant to PC54 and PC59 were inferred on ryegrass genome in proximity to RFLP marker CDO460, which was $\approx 3 \mathrm{cM}$ distant from RZ444 linked to QTL on LG3 of the MFB parent map (Fig. 1B and 2B).

Similarly, the map locations of Pc38, Prqlb, Pca cluster, and Pcq 2 were compared with QTL on LG7 of the MFA map. The hexaploid oat LG17 on which Pc38 and Prq1b are located $(16,30,37)$ is homoeologous with LG D of the Aah diploid oat population (38). The syntenic relationship between this diploid oat LG D and ryegrass LG7 has been known (33). Therefore, the equivalent locations of $P c 38$ and Prqlb were inferred on the ryegrass genome in proximity to RFLP marker CDO673, which was $\approx 6 \mathrm{cM}$ distant from BCD782 associated with QTL on the MFA parent LG7 (Figs. 1A and 2C). The Pca cluster and Pcq2 are located on LG A of the A. strigosa $\times$ A. wiestii $(A s w)$ diploid oat map $(24,42)$ and LG29 of the Ogle/MAM17-5 hexploid oat map $(43,44)$, respectively. RFLP marker BCD1280 linked to Pcq2 cosegregates with the isu2192 marker linked to Pca cluster (43). This BCD1280 is mapped on the Aah LG D that is homoeologous with the Asw LG A. Because a syntenic relationship was observed between this Ash LG D and ryegrass LG7 (33), QTL on LG7 of the MFA map also has potential for a counterpart of Pca cluster and Pcq2.

Genetic linkage between crown rust resistance and lignin biosynthetic genes. Five loci were detected by three RFLP probes corresponding to three lignin biosynthetic genes: two loci for $L p O M T 1$, two loci for $L p C A D 2$, and one locus for $L p C C R 1$ (data not shown). Of these, only four loci (LpOMT1-1, LpCAD21, LpCAD2-2, and LpCCR1) were mapped on LG7 in the 89progeny-based map, whereas LpOMT1-2 was not mapped on any LG (Fig. 3). The LpCAD2-1, LpCAD2-2, and LpCCR1 loci were at same location and $9 \mathrm{cM}$ distant from LpOMT1-1 (Fig. 3). Also, those loci were 8 and $17 \mathrm{cM}$, respectively, distant from BCD782 associated with crown rust QTL (Fig. 3). In the 152-progenybased map covering $16 \mathrm{cM}$ of LG7, those four loci were located at $0 \mathrm{cM}$ (LpOMT1-1), $8 \mathrm{cM}$ (LpCAD2-1), $9 \mathrm{cM}$ (LpCAD2-2), and $10 \mathrm{cM}$ (LpCCR1). The locus BCD782 was located at $16 \mathrm{cM}$.
LG 7

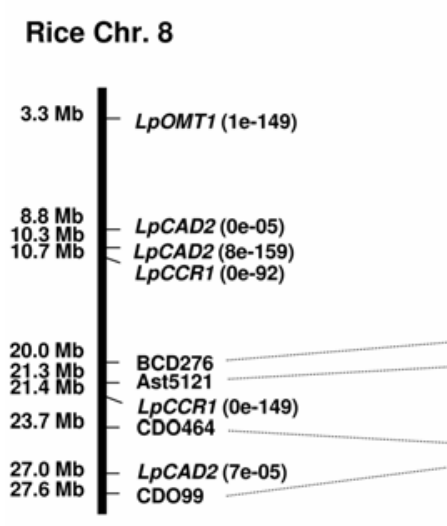

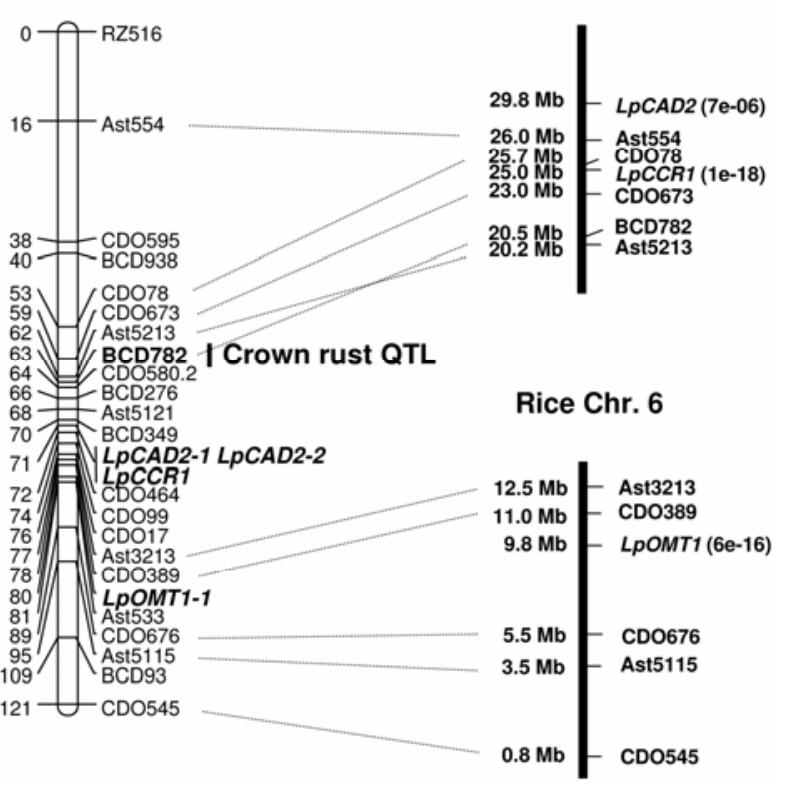

Fig. 3. Physical map locations of ortholoci on rice chromosomes 6 and 8 for three lignin genes and the RFLP markers on linkage group (LG) 7 of the integrated MFA/MFB genetic map constructed using only restriction fragment length polymorphism markers. The dotted lines connect ortholoci between ryegrass and rice. The putative duplication of three lignin genes on rice chromosomes is shown with $E$ value in parentheses after the gene names. 
Further analysis of ortholoci for those lignin genes and other RFLP markers on LG7 in the MFA map was conducted by blastn analysis using draft genome sequence of the grass model species, rice (Oryza sativa L.), available online from The Institute for $\mathrm{Ge}$ nomic Research (TIGR) (Rockville, MD). The putative ortholoci were located with high sequence similarity $(\leq E$ value $=7 \mathrm{e}-5)$ on rice chromosomes 6 and 8 , which are the syntenic counterparts of ryegrass LG7 (Fig. 3) (19,35). These ortholoci showed a high degree of colinearity between two species. In addition, rice chromosomes 6 and 8 showed multiple genomic regions for ortholoci of three lignin genes, LpOMT1, LpCAD2, and LpCCR1 (Fig. 3). According to our current comparative map resolution, it seems that the ortholocus of LpOMT1-1 is on rice chromosome 6 and the ortholoci of LpCAD2-1 and -2 as well as LpCCR1 are on rice chromosome 8, despite the putative ortholoci with higher sequence similarities that exist in other rice chromosomes.

\section{DISCUSSION}

Our results in this study indicate that a source of crown rust resistance might exist in Italian ryegrass cv. Floregon. Also, we observed a substantial variation in pustule size rather than presence or absence of pustules for crown rust over two locations and 2 years. Heagle and Moore (14) revealed that an oat cultivar with partial resistance to crown rust produced smaller pustules, fewer spores per pustule, and a longer period for sporulation. Therefore, the nature of crown rust resistance in our population could be partial resistance. In addition, the fairly high broad-sense heritability suggests that this resistance could be employed efficiently for improving crown rust resistance by selection in a ryegrass breeding program.

We detected four QTL from seven ratings at two locations for 2 years. Of them, QTL on LGs 2 and 7 were likely to be stable in diverse environmental conditions and, thus, have a potential for providing durable resistance to crown rust. Interestingly, the others on LGs 3 and 6 were derived from susceptible parent MFB. The existence of the QTL in this parent could be explained by a range of susceptibility for the MFB parent over seven ratings. The MFB parent showed moderate susceptibility under relatively low disease pressure and high susceptibility under relatively high disease pressure. Also, the transgressive segregation observed in progeny supports the idea that two QTL are present in the MFB parent; however, their effects could be too small to show a high level of resistance to crown rust. Furthermore, these QTL may be associated with specific environmental conditions such as high disease pressure because they were detected by only the second rating at the OJN plot or one rating at the SIU plot in 2004. In fact, the phenotypic distributions of these two ratings were highly skewed toward susceptible reaction.

Also, it is possible that the QTL on LG 6 of the MFB map is race specific because it was detected only in the SIU plot. Crown rust is a fungus that can develop different physiological races. More than 290 physiological races of oat crown rust ( $P$. coronata f. sp. avenae) have been reported (27) and evidence of existence of the races in ryegrass crown rust $(P$. coronata $\mathrm{f}$. $\mathrm{sp}$. lolli) has been found $(6,31,41)$. Although race specificity of crown rust on ryegrass is a likely phenomenon, major genes for resistance to specific races do not play a major role in resistance of ryegrass cultivars to crown rust (41). Resistance to crown rust appears to be polygenic or oligogenic, in which most genes confer partial resistance with a certain degree of additivity among resistance loci $(40,41)$. Rapid and effective specialization of crown rust on ryegrass genotypes severely limits the effectiveness and durability of resistance genes with major effects $(39,41)$. The QTL from the parent MFB needs to be studied in greater detail to determine its gene action and potential for interaction with the pathogen.

The map locations of QTL identified in this study were compared with those of QTL for crown rust resistance identified in different ryegrass mapping populations via comparative mapping approach. Out of two known QTL, LpPc1 and LpPc3 on LG2, $L p P c 1$ is likely to be a conserved resistance gene for crown rust because it was detected from two different ryegrass populations $(10,29)$. Therefore, it is possible that the QTL on LG2 of the MFA map in this study corresponds to $L p P c 1$, even though the RFLP marker linked to this QTL was $\approx 38 \mathrm{cM}$ distant from the RFLP markers linked to $L p P c 1$. Furthermore, the map distance between markers could be under- or overestimated due to missing data of the markers. In fact, the marker data of BCD1184 linked to this QTL includes two missing points out of 89 progeny. Simultaneously, we could not exclude the possibility that this QTL corresponds to $L p P c 3$ because the current map resolution provided no information on the relative distance between our QTL and $L p P c 3$.

Three additional QTL on LGs 3, 6, and 7 detected in this study appeared to be unique ryegrass QTL because no corresponding QTL have been identified in other ryegrass-mapping populations. There are three possible explanations for detecting these unique QTL. First, the interspecific population used in this study may contain unique genes for crown rust resistance which are derived from Italian ryegrass because the previous mapping studies $(10,29,35)$ detected crown rust resistance genes using perennial ryegrass populations. Second, the additional QTL detected in this study could be associated with field resistance to crown rust, because the previous studies were conducted under growth chamber or greenhouse conditions with artificial inoculation, whereas this study was performed under natural field conditions. Portyanko et al. (30) indicated the existence of discrepancies between greenhouse and field tests for detecting QTL for crown rust resistance in oat. Last, it may be due to a lack of phenotypic contrast between the parents of the other ryegrass-mapping populations for the loci in question.

The syntenic relationships of the QTL on LGs 3 and 7 relative to oat crown rust resistance genes suggest that the ortholoci for crown rust resistance might be present between ryegrass and oat. In fact, perennial ryegrass genotypes resistant to $P$. coronata $\mathrm{f}$. sp. lolii are also generally resistant to $P$. coronata f. sp. avenae (11), indicating that there is considerable cross-resistance of crown rust between two grass species. The presence of orthologous resistance genes for a pathogen among related grass species has been known previously. For blast disease (Magnaporthe grisea), Chen et al (5) and Curley et al. (8) identified ortholoci for blast resistance genes between rice and barley (Hordeum vulgare L.) and ryegrass, respectively.

Constitutive resistance, based on chemical or enzymatic modifications to the host plant, may be a mechanism to develop broad resistance or non-race-specific resistance to crown rust in ryegrass (34). A loss of crown rust resistance following selection for low lignin concentration in smooth bromegrass suggests that lignin may be a mechanism of constitutive resistance to crown rust (9). Within current map resolution, the QTL on LG7 likely was distant from the loci for three key lignin genes (LPOMT1, $L p C A D 2$, and $L p C C R 1$ ). This indicates that a negative correlation between two traits, crown rust resistance and lignin content, might be easily broken in ryegrass. However, this conclusion should be treated with caution because of a number of QTL for herbage quality traits associated with those lignin loci on LG7 in the p150/112 ryegrass reference map (7). The relationship between these herbage quality QTL and crown rust QTL still remains unknown.

In addition, other genes or the duplicated loci for OMT and $\mathrm{CAD}$ which were not employed in the current map might be associated with either the QTL on LG7 or on other LGs. In fact, three genes have been identified for OMT and CAD in perennial ryegrass $(15,24)$. Further, two of three CAD genes ( $L p C A D 1$ and $L p C A D 2$ ) have been known as multiple-copy genes (24). The genetic mapping study of Cogan et al. (7) also detected three RFLP loci for $L p C A D 2$. One of them was closely located with both 
LpOMT1 and LpCCR1 loci on LG7. The other LpCAD2 locus was mapped on LG2. Our study detected two RFLP loci for both LpOMT1 and LpCAD2. One LpOMT1 locus and two LpCAD2 loci were mapped with one LpCCRI locus on LG7. Therefore, the genetic linkage between crown rust resistance and lignin content should be studied further.

In summary, the present study identified four QTL for crown rust resistance in an interspecific ryegrass population under diverse natural field conditions. Comparative QTL mapping found that these QTL, except one on LG2, were different from the QTL previously identified in the perennial ryegrass populations evaluated under growth chamber and greenhouse conditions, indicating the existence of a new source of crown rust resistance in our ryegrass population. If the RFLP markers linked to the QTL are converted into PCR-based molecular markers, marker-assisted selection using the PCR markers can be used efficiently to accumulate multiple crown rust resistant genes into an elite ryegrass cultivar. Furthermore, this study suggests that comparative mapping can be an effective method for understanding a genetic system of crown rust resistance to different formae speciales of the pathogen between ryegrass and oat.

\section{ACKNOWLEDGMENTS}

All plant materials, including the mapping population used in this study, were provided by R. Barker, United States Department of Agriculture-Agricultural Research Service, Corvallis, OR.

\section{LITERATURE CITED}

1. Alm, V., Fang, C., Busso, C. S., Devos, K. M., Vollan, K., Grieg, Z., and Rognli, O. A. 2003. A linkage map of meadow fescue (Festuca pratensis Huds.) and comparative mapping with other Poaceae species. Theor. Appl. Genet. 108:25-40.

2. Arahana, V. S., Graef, G. L., Specht, J. E., Steadman, J. R., and Eskridge, K. M. 2001. Identification of QTL for resistance to Sclerotinia sclerotiorum in soybean. Crop Sci. 41:180-188.

3. Bush, A. L., and Wise, R. P. 1996. Crown rust resistance loci on linkage groups 4 and 13 in cultivated oat. J. Hered. 87:427-432.

4. Chakraborty, N., Bae, J., Warnke, S., Chang, T., and Jung, G. 2005. Linkage map construction in allotetraploid creeping bentgrass (Agrostis stolonifera L.). Theor. Appl. Genet. 111:795-803.

5. Chen, H., Wang, S., Xing, Y., Xu, C., Hayes, P. M., and Zhang, Q. 2003. Comparative analyses of genomic locations and race specificities of loci for quantitative resistance to Pyricularia grisea in rice and barley. Proc. Natl. Acad. Sci. USA 100:2544-2549.

6. Clark, R. G., Villata, O. N., and Hepworth, G. 1997. Evaluation of resistance to five isolates of Puccinia coronata f. sp. lolii in 19 perennial ryegrass cultivars. Aust. J. Agric. Res. 48:191-198.

7. Cogan, N. O. I., Smith, K. F., Yamada, T., Francki, M. G., Vecchies, A. C., Jones, E. S., Spangenberg, G. C., and Forster, J. W. 2005. QTL analysis and comparative genomics of herbage quality traits in perennial ryegrass (Lolium perenne L.). Theor. Appl. Genet. 110:364-380.

8. Curley, J., Sim, S. C., Warnke, S., Leong, S., and Jung, G. 2005. QTL mapping of resistance to gray leaf spot in ryegrass. Theor. Appl. Genet. 111:1107-1117

9. Delgado, N. J., Casler, M. D., Grau, C. R., and Jung, H. G. 2002. Reactions of smooth bromegrass clones with divergent lignin or etherified ferulic acid concentration to three fungal pathogens. Crop Sci. 42:18241831

10. Dumsday, J. L., Smith, K. F., Forster, J. W., and Jones, E. S. 2003. SSRbased genetic linkage analysis of resistance to crown rust (Puccinia coronata f. sp. lolii) in perennial ryegrass (Lolium perenne). Plant Pathol. 52:628-637.

11. Eshed, N., and Dinoor, A. 1980. Genetics of pathogenicity in Puccinia coronata; pathogenic specialization at the host genus level. Phytopathology 70:1042-1046.

12. Hayward, M. D. 1977. Genetic control of resistance to crown rust (Puccinia coronata Corda) in ryegrass (Lolium perenne L.) and its implication in plant breeding. Theor. Appl. Genet. 51:49-53.

13. Hayward, M. D., McAdam, N. J., Jones, J. G., Evans, C., Evans, G.. M., Forster, J. W, Ustin, A., Hussain, K. G., Quader, B., Stammers, M., and Will, J. A. K. 1994. Genetic markers and the selection of quantitative traits in forage grasses. Euphytica 77:269-275

14. Heagle, A. S., and Moore, M. B. 1970. Some effects of moderate adult resistance to crown rust of oat. Phytopathology 60:461-466.

15. Heath, R., Huxley, H., Stone, B., and Spangenberg, G. 1998. cDNA cloning and differential expression of three caffeic acid $O$-methyltransferase homologues from perennial ryegrass (Lolium perenne L.). J. Plant Physiol. 153:649-657.

16. Irigoyen, M. L., Loarce, Y., Fominaya, A., and Ferrer, E. 2004. Isolation and mapping of resistance gene analogs from the Avena strigosa genome. Theor. Appl. Genet. 109:713-724.

17. Jansen, R. C. 1993. Interval mapping of multiple quantitative trait loci. Genetics 135:205-211.

18. Jansen, R. C., and Stam, P. 1994. High resolution of quantitative traits into multiple loci via interval mapping. Genetics 136:1447-1455.

19. Jones, E. S., Mahoney, N. L., Hayward, M. D., Armstead, I. P., Jones, J. G., Humphreys, M. O., King, I. P., Kishida, T., Yamada, T., Balfourier, F., Charmet, G., and Forster, J. W. 2002. An enhanced molecular marker based genetic map of perennial ryegrass (Lolium perenne) reveals comparative relationships with other Poaceae genomes. Genome 45:282295.

20. Jones, S., Dupal, P., Dumsday, L., Hughes, J., and Forster, W. 2002. An SSR-based genetic linkage map for perennial ryegrass (Lolium perenne L.). Theor. Appl. Genet. 105:577-584.

21. Kimbeng, C. A. 1999. Genetic basis of crown rust resistance in perennial ryegrass, breeding strategies, and genetic variation among pathogen populations: a review. Aust. J. Exp. Agric. 39:361-378.

22. Kopec, D. M., Funk, C. R., and Haliski, P. M. 1983. Sources and resistance to crown rust within perennial ryegrass. Plant Dis. 67:98-100.

23. Kremer, C. A., Lee, M., and Holland, J. B. 2001. A restriction fragment length polymorphism based linkage map of a diploid Avena recombinant inbred line population. Genome 44:192-204.

24. Lynch, D., Lidgett, A., McInnes, R., Huxley, H., Jones, E., Mahoney, N., and Spangenberg, G. 2002. Isolation and characterization of three cinnamyl alcohol dehydrogenase homologue cDNAs from perennial ryegrass (Lolium perenne L.). J. Plant Physiol. 159:653-660.

25. McInnes, R., Lidgett, A., Lynch, D., Huxley, H., Jones, E., Mahoney, N., and Spangenberg, G. 2002. Isolation and characterization of a cinnamoylCoA reductase gene from perennial ryegrass (Lolium perenne L.). J. Plant Physiol. 159:415-422.

26. McVeigh, K. J. 1975. Breeding for Resistance to Crown Rust (Puccinia coronata Corda var. lolii Brown) in Turf Type Perennial Ryegrass (Lolium perenne). Rutgers University, New Brunswick, NJ.

27. Michel, L. J., and Simons, M. D. 1977. Aggressiveness and virulence of Puccinia coronata avenae isolates, 1971-1975. Plant Dis. Rep. 61:621625.

28. Moerschbacher, B. M., Noll, U., Gorrichon, L., and Reisener, H. J. 1990. Specific inhibition of lignification breaks hypersensitivity resistance of wheat to stem rust. Plant Physiol. 93:465-470.

29. Muylle, H., Baert, J., Van Bockstaele, E., Pertijs, J., and Roldán-Ruiz, I. 2005. Four QTLs determine crown rust (Puccinia coronata f. sp. lolii) resistance in a perennial ryegrass (Lolium perenne) population. Heredity 95:348-357.

30. Portyanko, V. A., Chen, Q., Rines, H. W., Phillips, R. L., Leonard, K. J., Ochocki, G. E., and Stuthman, D. D. 2005. Quantitative trait loci for partial resistance to crown rust, Puccinia coronata, in cultivated oat, Avena sativa L. Theor. Appl. Genet. 111:313-324.

31. Potter, L. R., Cagas, B., Paul, V. H., and Birckenstaedt, E. 1990. Pathogenicity of some European collections of crown rust (Puccinia coronata Corda) on cultivars of perennial ryegrass. Phytopathology 130:119126.

32. Reheul, D., and Ghesquiere, A. 1996. Breeding perennial ryegrass with better crown rust resistance. Plant Breed. 115:465-469.

33. Sim, S., Chang, T., Curley, J., Warnke, S. E., Barker, R. E., and Jung, G. 2005. Chromosomal rearrangements differentiating the ryegrass genome from the Triticeae, oat, and rice genomes using common heterologous RFLP probes. Theor. Appl. Genet. 110:1011-1019.

34. Takahashi, W., Fujimori, M., Miura, Y., Komatsu, T., Nishizawa, Y., Hibi, T., and Takamizo, T. 2005. Increased resistance to crown rust disease in transgenic Italian ryegrass (Lolium multiflorum Lam.) expressing the rice chitinase gene. Plant Cell Rep. 23:811-818.

35. Thorogood, D., Paget, M., Humphreys, M., Turner, L., Armstead, I., and Roderick, H. 2001. QTL analysis of crown rust resistance in perennial ryegrass-implications for breeding. Int. Turf. Soc. Res. J. 9:218-223

36. Warnke, S. E., Barker, R. E., Jung, G., Sim, S. C., Rouf Mian, M. A., Saha, M. C., Brilman, L. A., Dupal, M. P., and Forster, J. W. 2004. Genetic linkage mapping of an annual x perennial ryegrass population. Theor. Appl. Genet. 109:294-304.

37. Wight, C. P., O'Donoughue, L. S., Chong, J., Tinker, N., and Molnar, S. J. 2004. Discovery, localization, and sequence characterization of molecular markers for the crown rust resistance genes $P c 38, P c 39$, and Pc48 in cultivated oat (Avena sativa L.). Mol. Breed. 14:349-361. 
38. Wight, C. P., Tinker, N. A., Kianian, S. F., Sorrells, M. E., O'Donoughue, L. S., Hoffman, D. L., Groh, S., Scoles, G. J., Li, C. D., Webster, F. H., Phillips, R. L., Rines, H. W., Livingston, S. M., Armstrong, K. C., Fedak, G., and Molnar, S. J. 2003. A molecular marker map in 'Kanota' $\times$ 'Ogle' hexaploid oat (Avena spp.) enhanced by additional markers and a robust framework. Genome 46:28-47.

39. Wilkins, P. W. 1975. Implications of host-pathogen variation for resistance breeding in the grass crop. Ann. Appl. Biol. 81:257-261.

40. Wilkins, P. W. 1975. Inheritance of resistance to Puccinia coronata Corda and Rhynchosporium orthosporum Caldwell in Italian ryegrass. Euphytica 24:191-196.
41. Wilkins, P. W. 1978. Specialization of crown rust on highly and moderately resistant plants of perennial ryegrass. Ann. Appl. Biol. 88:179184.

42. Wise, R. P., Lee, M., and Rayapati, P. J. 1996. Recombination within a 5centimorgan region in diploid Avena reveals multiple specificities conferring resistance to Puccinia coronata. Phytopathology 86:340-346.

43. Zhu, S., and Kaeppler, H. F. 2003. Identification of quantitative trait loci for resistance to crown rust in oat line MAM17-5. Crop Sci. 43:358-366.

44. Zhu, S., Leonard, K. J., and Kaeppler, H. F. 2003. Quantitative trait loci associated with seedling resistance to isolates of Puccinia coronata in oat. Phytopathology 93:860-866. 